WS6-P01

\title{
Effects of the Trace Interval on Surface Wave Dispersion and Inversion
}

T.J. Gong* (BGP,CNPC), H.L. Chen (BGP,CNPC), X.D. Li (BGP,CNPC), Y.Q. Ye (BGP,CNPC), J. Liu (BGP,CNPC), H.J. Zhang (BGP,CNPC), C.Z. Ni (BGP, CNPC), G.D. Ding (BGP,CNPC) \& B.J. Liang (BGP,CNPC)

\section{SUMMARY}

It is the trace interval that determines the spatial frequency property of one shot gather. As the trace interval increases, spatial aliasing emerges and data quality deteoriates. Aliased surface waves in the dispersion energy map have false higher-mode dispersion curves, or low velocity noise, and have missing or pronged dispersion energy trends at certain frequency ranges. Qualitatively, large spacing causes a severe aliasing that smears the dispersion plot, yielding it unusable. As long as spatial aliasing is not dominant, the surface waves can be used to invert the corresponding subsurface structure. We present a synthetic example to test the effectiveness of the high resolution linear Radon transform (HRLRT) and the least squares Radon transform (LSRT), which are used to calculate dispersion curves. Since the HRLRT typically affords a better low frequency response, we use it to map the field example. Effects caused by different spatial intervals in dispersion maps of synthetic and field examples are also shown. A genetic algorithm is used in the inversion to determine the subsurface structure, and these results are then compared with a nearby borehole test. The agreement between the test and the model shows the promising prospect of the surface wave methods. 


\section{Introduction}

Surface waves are a major form of destructive noise with strong amplitude, and geophysicists have been endeavouring to remove them from shot gathers in reflection seismology. However, owing to the dispersive properties of this wave type, they have been widely used as a tool for near surface-structure investigations in recent years, and are drawing more and more attention. One prevailing analysis method is the multichannel analysis of surface waves (MASW), initiated and developed by the Kansas Geological Survey. Several examples of applications and studies amongst a long list can be found in published work (Miller et al., 1999; Xia et al., 1999a, b, 2000; Park et al., 2000, 2005). Previous studies have shown that higher frequency parts of surface waves have a higher sensitivity to shallow strata, while lower frequency parts have deeper penetration ability. Higher mode data has a deeper investigation depth than the fundamental mode data, and higher mode data increases the resolution of inverted S-wave velocities (Xia et al., 2000). Commonly used intervals are $1 \mathrm{~m}, 2 \mathrm{~m}$ or $5 \mathrm{~m}$, and the total spread consists of 24,40 or 60 traces, depending on the scale and depth of the target zone. An approximate prospecting thickness of surface waves is limited within a wavelength (Socco et al., 2010). Park et al. (2001) showed that with a given number of channels available, a greater receiver spacing is preferred for higher-resolution dispersion curve imaging, as long as a spatial aliasing problem does not occur. It is for this reason that we have been inspired to begin a thorough study to investigate the receiver spacing and aliasing issue. Study results show that as long as the spatial aliasing problem does not affect the phase velocity picking, we can make use of the dispersion property.In this article, we try to analyse the dispersion properties of the surface waves extracted from common shot gathers (CSG) with larger trace spacing. We started with a synthetic model comprised of two modes after a brief discussion of Nyquist's Sampling Theorem, to test the high-resolution linear Radon transform (HRLRT) (Luo et al., 2008), and the least-squares Radon transform (LSRT) methods for mapping the dispersion property. The similarities of the results with the theoretical curves validate the accuracy of the two methods. The surface waves extracted from the CSG are shown afterwards, and the inversion is conducted based on the dispersion curves calculated by HRLRT with a genetic algorithm (Pezeshk and Zarrabi, 2005). Inversion results compared with a nearby borehole test show a good agreement.

\section{Theoretical Formulations}

The temporal and spatial sample theorem can be stated as: $f_{N}=1 /(2 * \Delta t), k_{N}=1 /(2 * \Delta x)$, where $f_{N}$ is the Nyquist frequency, since $\Delta t$ can be $1 \mathrm{~ms}$ or smaller, $f_{N}$ can reach $500 \mathrm{~Hz}$ or higher, which is way outside the surface waves' frequency range - from several to tens of Hertz. For this part, we barely encounter a problem. And $k_{N}$ is the Nyquist wavenumber that can cause aliasing when the trace interval $\Delta x$ is bigger than half of the wavelength $\lambda$. We need

$$
\lambda=v / f>2 * \Delta x
$$

From relationship $v=f / k$, we can see if $\mathrm{k}$ is outside the normal part of the wavenumber range, i.e. the Nyquist wavenumber, velocity will correspondingly take on an aliasing property. Specific examples will be used to illustrate the effects of aliasing.

\section{Theoretical Example}

We use a synthetic example of surface waves to test the dispersion curve extraction methods, the LSRT and HRLRT (see

Figure 1 (a-d)). Spacing for the total 45 traces is $2 \mathrm{~m}$, and the minimum offset $10 \mathrm{~m}$. Comparing Figure 1(c) and (d), one can notice that both the LSRT and HRLRT show a similar trend of dispersion as the modeled dispersion curves (b) within the frequency range of $20-100 \mathrm{~Hz}$, which validate the accuracy of the methods, but the latter one exhibits a better focusing power. In the following field example, we use the HRLRT to map the dispersion energy plot.

Figure 1(e) - (h) show calculated dispersion plots for $4 \mathrm{~m}, 8 \mathrm{~m}, 16 \mathrm{~m}$ and $32 \mathrm{~m}$, respectively. With longer intervals come more serious aliasing phenomena, expanding from higher frequency into lower frequency. Note that in spite of aliasing, the dispersion trend can be discerned by its relatively higher 


\section{Amsterdam '14}

energy. For this example, if we want to estimate the model, the maximum trace spacing should be no larger than $16 \mathrm{~m}$ with no additional processing, such as spatial interpolation.

(a)
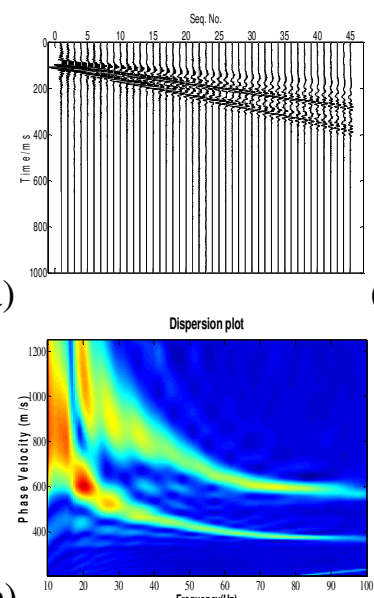

(b)
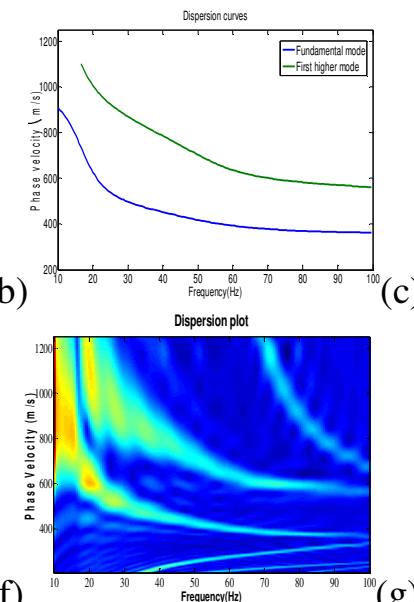

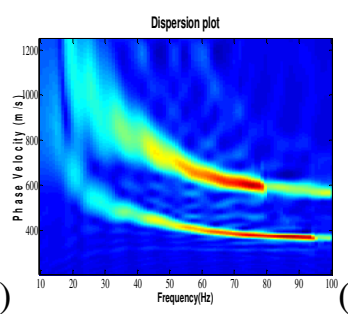

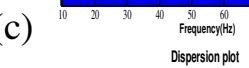

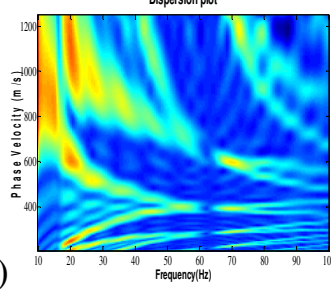

(d)

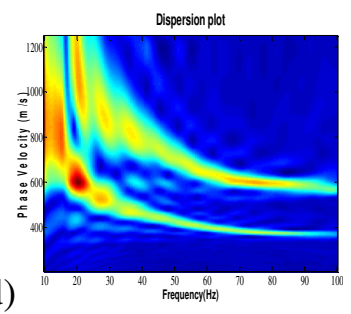

(g)

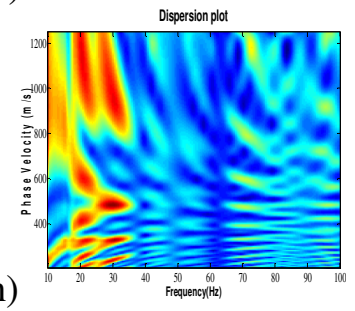

Figure 1 (a) Synthetic surface waves; (b) Fundamental mode and higher mode of the synthetic example; (c) Dispersion energy with LSRT in f-v domain for $2 \mathrm{~m}$ spacing; (d) Same as (c) with HRLRT;(e) HRLRT for $4 \mathrm{~m}$ spacing; (f) HRLRT for $8 \mathrm{~m}$; (g) HRLRT for $16 \mathrm{~m}$; (h) HRLRT for $32 \mathrm{~m}$.

According to Equation (1), the maximum frequency of the fundamental mode dispersion curve without aliasing for $16 \mathrm{~m}$ spacing is $\sim 19 \mathrm{~Hz}$, and $\sim 27 \mathrm{~Hz}$ for the first higher mode. The high-end frequency can be as high as $48-50 \mathrm{~Hz}$, even with the weak false energy trend caused by the aliasing. We conclude that as long as the aliasing does not overwhelm the energy plot, the dispersion properties can still be extracted and analyzed.

From this experiment, we can see that there are two characteristics for the aliasing: first, it mainly occurs at the higher frequency parts in two different forms with abnormally low or high wavenumbers, which leads to a higher-mode like dispersion curve (top right of

Figure 1(f)), or low velocity noise (bottom right of

Figure 1(f)), respectively. Second, the dispersion energy trends are missing or pronged at certain frequency ranges (

Figure $1(\mathrm{~g}, \mathrm{~h})$ ), which are caused by destructive interference of the aliasing with the normal part.

\section{Field Example}

Common shot gathers were acquired in the DaGang Oilfield, Cangzhou District, in the winter of 2012. Only one $10 \mathrm{~Hz}$ geophone was planted at one receiver station without any array, in order to preserve the property of the surface waves. The spacing was set at $10 \mathrm{~m}$. The surface waves extracted from the CSP are given in

Figure 2. The extraction is accomplished in a module called GrndRolAtten in GeoEast ${ }^{\circledR}$. The method is a time-frequency analysis process. With differences given by user-defined frequency attributes, velocity ranges and energy threshold between reflection and ground roll waves, the latter is detected and analyzed in both the temporal and spatial domain for its own attenuation.

We took the dispersion plots of the first 30 traces in the extracted CSP, with $10 \mathrm{~m}, 20 \mathrm{~m}, 30 \mathrm{~m}$ and 40 $\mathrm{m}$ trace intervals, as shown in Figure 3 (a-d). For the raw $10 \mathrm{~m}$ interval case (Figure 3(a)), three different modes can be defined from 4 to $12 \mathrm{~Hz}$, together with a possible noisy aliasing zone at around $6 \mathrm{~Hz}$. For this area, the energy focus is not as clear-cut as the other parts, thus we discard it in dispersion curve picking. When the interval is doubled, (Figure 3(b)), the second highest mode becomes pronged at the lower end. The top right panel shows a vague emergence of the aliasing. If the spacing is tripled (Figure 3(c)), the aliasing is much more conspicuous; the upper half has a stronger energy portion and causes inaccurate mode separation. If the spacing is quadrupled, (Figure $3(d)$ ), mode separation is nearly impossible. 


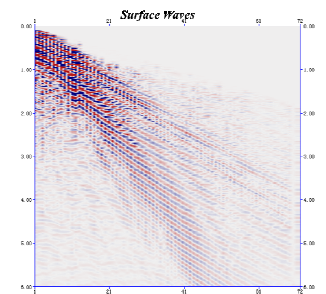

Figure 2 Surface waves extracted from CSP. Horizontal axis is Seq. \#, vertical axis is time in seconds.

If we halve the distance between adjacent traces, a much clearer dispersion image can be obtained (Figure 3(e)), using an interpolation method after Spitz (1991). For this case, it is obvious that the best trace interval should be a little less than $10 \mathrm{~m}$. But based on the variations of the dispersion plots as the trace interval increases, we can see that so long as the aliasing energy is not predominant to an extent enough to be misleading $(\leq 30 \mathrm{~m})$, dispersion properties can still be extracted and analyzed.

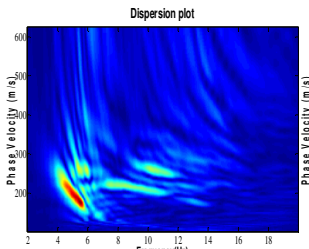

(a)

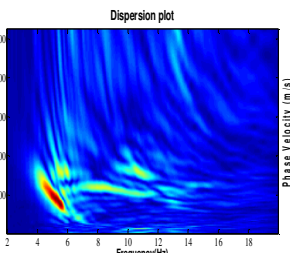

(b)

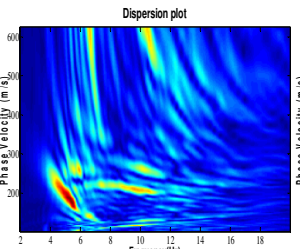

(c)

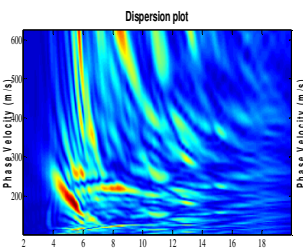

(d)

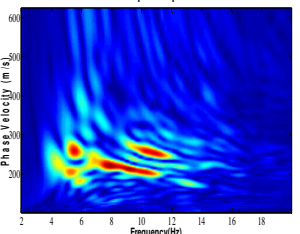

(e)

Figure 3 Dispersion plot for (a) $10 \mathrm{~m}$ interval; (b) $20 \mathrm{~m}$; (c) $30 \mathrm{~m}$; (d) $40 \mathrm{~m}$; and (e) interpolated $5 \mathrm{~m}$.

We adopted a two-phase process in the inversion of the subsurface structure with a genetic algorithm (Gong et al., 2012), using the dispersion curves picked from the raw $10 \mathrm{~m}$ interval:

- Phase 1: Inversion with the fundamental mode for a rough estimate of the possible earth structure.

- Phase 2: Inclusion of the higher modes for estimating a more accurate result.

(a)
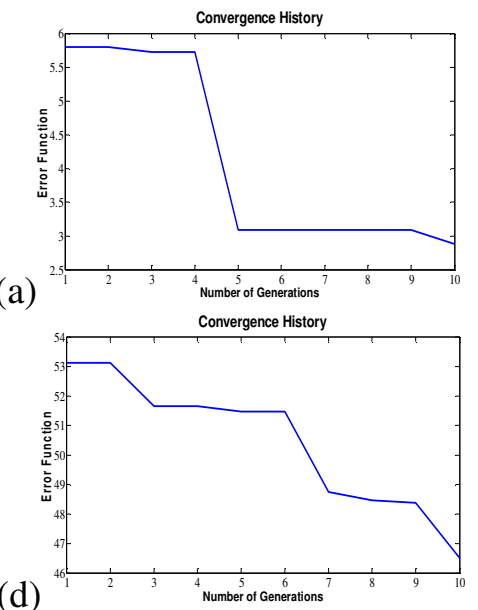

(b)

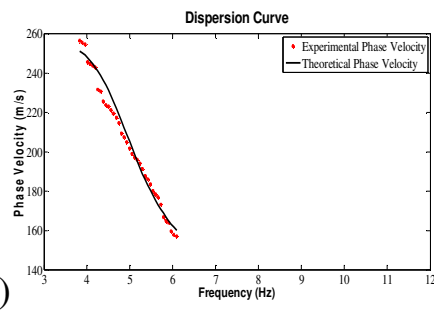

(e)

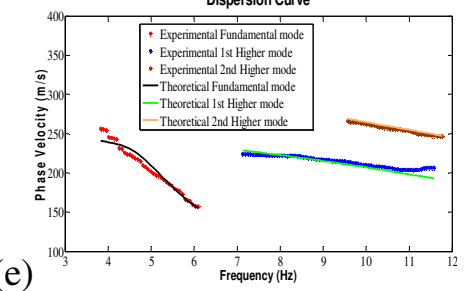

(c)

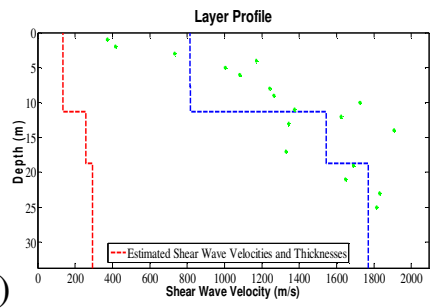

(f)

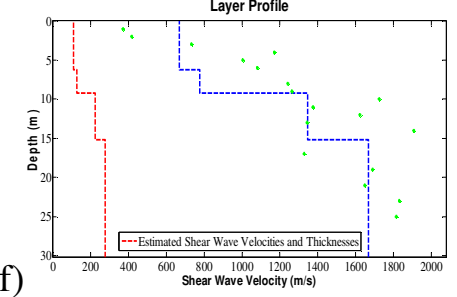

Figure 4 Two-phase inversion process. Phase 1 (a-c) for fundamental mode case: (a) Convergence history; (b) Dispersion curves comparison; (c) Inversion and borehole results comparison. Red dashed line is estimated Vs and thickness, blue dashed line is the corresponding Vp, green scatters are borehole results. Phase 2 (d-f) for multi-mode case: same symbol indications as in (a-c) are used.

A micro-logging test was conducted near the shot point around the $12^{\text {th }}$ sequence number, with an offset of about $120 \mathrm{~m}$ to the southeast. The test results are shown in the inversion comparisons. The fundamental mode-only inversion results together with the borehole results can be seen in Figure 4 (ac). The results reveal that the deeper parts of the inversion agree well with the borehole results, while the first few meters near the surface show a noticeable discrepancy. This is easy to understand, since lower frequencies have a greater sensitivity to imaging deeper layers, and the higher frequencies are 


\section{Amsterdam '14}

closely related to the shallower layer. Thus, the latter's loss affects the accuracy of the inversion of the shallow parts. The multi-mode case inversion results are shown with the borehole results in Figure 4 (d-f). The overall velocity trend is the same as in the fundamental case. But for 0-10 m, the multimode case inversion has a better vertical resolution with two layers than the one layer interpretation for the fundamental case. The borehole results were calculated from the first breaks picked from the micro-logging record, whose errors explain the differences between adjacent velocity scatters.

\section{Conclusions}

Spatial aliasing occurs when the trace spacing increases, and the frequency-velocity energy map is distorted. The larger the spacing, the more serious the distortion. As long as the spatial aliasing energy is not overwhelming, dispersion properties can be extracted and analyzed for further structural estimation. The useful high-end frequency can be outside the Nyquist frequency. Inclusion of higher frequencies and modes in the inversion calculation will enhance the accuracy and resolution of the inversion of shallower levels. Strategies that can be used to attenuate the influence of spatial aliasing, such as de-aliasing interpolation, can maximize the amount of useful information acquired from surface waves in large-spacing data acquisition.

\section{Acknowledgements}

We wish to thank BGP, CNPC for permission to publish the results. Special thanks go to Professor Mauricio Dino Sacchi for his kind help in programming. The constructive comments from Christian Manthei are greatly appreciated.

\section{References}

Gong, T., Liu, M., Zhang, H., Li, X., Chen, H., Liu, J., Liu, R. and Ye, Y. [2013] Near-surface structure estimation using Rayleigh wave and a genetic algorithm. First Near Surface Geophysics Asia Pacific Conference, Beijing, July, Expanded Abstracts.

Luo, Y., Xia, J., Miller, R. D., Xu, Y., Liu, J. and Liu, Q. [2008] Rayleigh-wave dispersive energy imaging by high resolution linear Radon transform. Pure and Applied Geophysics, 165(5), 903-922.

Miller, R.D., Xia, J., Park, C.B. and Ivanov, J. [1999] Multichannel analysis of surface waves to map bedrock. The Leading Edge, 18, 1392-1396.

Park, C.B., Miller, R.D. and Xia, J. [1999] Multi-channel analysis of surface waves (MASW). Geophysics, 64, 800-808.

Park, C.B., Miller, R.D. and Xia, J. [2001] Offset and resolution of dispersion curve in multichannel analysis of surface waves (MASW). Proceedings of SAGEEP, Expanded Abstracts.

Park, C.B., Miller, R.D., Xia, J., Ivanov, J., Sonnichsen, G.V., Hunter, J.A., Good, R L., Burns, R.A. and Christian, H. [2005] Underwater MASW to evaluate stiffness of water-bottom sediments. The Leading Edge, July, 724-728.

Pezeshk, S. and Zarrabi, M. [2005] A new inversion procedure for spectral analysis of surface waves using a genetic algorithm. Bulletin of the Seismological Society of America, 95(5), 1801-1808.

Socco, L.V., Foti, S. and Boiero, D. [2010] Surface-wave analysis for building near-surface velocity models-established approaches and new perspectives. Geophysics, 75(5), 75A83-75A102.

Spitz, S. [1991] Seismic trace interpolation in the F-X domain. Geophysics, 56(6), 785-794.

Xia, J., Miller, R.D. and Park, C.B. [1999a] Estimation of near-surface shear-wave velocity by inversion of Rayleigh wave. Geophysics, 64, 691-700.

Xia, J., Miller, R.D., Park, C.B., Hunter, J.A. and Harris, J.B. [1999b] Evaluation of the MASW technique in unconsolidated sediments. $69^{\text {th }}$ Annual International Meeting, SEG, Expanded Abstracts, 437-440.

Xia, J., Miller, R.D. and Park, C.B. [2000] Advantages of calculating shear-wave velocity from surface waves with higher modes. $70^{\text {th }}$ Annual International Meeting, SEG, Expanded Abstracts, 1295-1298. 